\title{
Intrahepatic cholestasis of pregnancy
}

INSERM

\section{Source}

INSERM. (1999). Orphanet: an online rare disease and orphan drug data base.

Intrahepatic cholestasis of pregnancy. ORPHA:69665

Intrahepatic cholestasis of pregnancy (ICP) is a cholestatic disorder characterized by (i) pruritus with onset in the second or third trimester of pregnancy, (ii) elevated serum aminotransferases and bile acid levels, and (iii) spontaneous relief of signs and symptoms within two to three weeks after delivery. 